\title{
Analysis of Core Competency Literacy of Innovative Technology Talents in Intelligent Robot Industry
}

\author{
Fang Yu-Shen ${ }^{1, a}$, Wang Shi-Jie ${ }^{1, b^{*}}$, Hu Run-Jiao ${ }^{1}$, Zhou Zhao-Hong ${ }^{1}$, Zhu Bi-Yun ${ }^{1}$ \\ ${ }^{1}$ Economics and Management College of Zhaoqing University, Zhaoqing, Guangdong, China \\ afang.yushen@qq.com \\ b*miroslav.klose.m@foxmail.com
}

\begin{abstract}
According to intelligent robots are a holistic and interdisciplinary field of work, what is needed is not only the performance of individual capabilities but also the performance of educated talents, to become an advantageous source of industrial competition. To strengthen the professional competence of talents in this industry, this research aims to develop the functional qualities of the key professions of "innovative scientific and technological talents" as a reference for the development of subsequent function-oriented training courses. The function is to complete the task of a specific occupation (or category), and the required combination of capabilities can be used by enterprise organizations in selecting, using, cultivating and retaining talents, and for universities to refer to when they develop professional courses. This research takes the experts and supervisors of the intelligent robot industry in the Guangdong-Hong Kong-Macao Greater Bay Area as the research object, and constructs a blueprint for the functional literacy of "innovative technology talents". Obtaining six key functional literacy groups and 38 basic functional elements, as well as the knowledge, skills, and attitude required by the profession, can also become an important benchmark for vocational training, to accelerate the cultivation of talent in the intelligent robot industry, gradually improving the human structure, and create Industrial value chain.
\end{abstract}

Keywords: Human resource development, Innovative scientific and technological talent, Competence model,

Smart robot, Guangdong-Hong Kong-Macao greater bay area

\section{智能机器人产业创新科技人才核心职能素养分析}

\author{
方㻦绅 $1, \mathrm{a}$ 王仕杰 ${ }^{2, \mathrm{~b}^{*}}$ 胡润姣 ${ }^{3}$ 周照红 ${ }^{4}$ 朱碧云 ${ }^{5}$ \\ $1,2,3,1,5$ 肇庆学院经济与管理学院, 肇庆市, 广东省, 中国 \\ afang.yushen@qq.com \\ b*miroslav.klose.m@foxmail.com
}

\section{摘要}

鉴于智能机器人是一种整体及跨学科领域的工作组合, 需要的不仅是个体能力表现, 更需要教育人才 的表现, 才能成为产业竞争的优势来源, 为强化该产业人才的专业能力素质, 本研究旨在发展” 创新 科技人才”关键职业之职能素养，以作为后续之职能导向训练课程发展的参考。职能为完成特定职业 (或职类)工作任务, 所需具备的能力组合, 可以供企业组织在选才、用才、育才及留才, 和供高校在 开发专业课程时参考指引。爰本研究以粤港澳大湾区智能机器人产业专家及主管职为研究对象, 建构” 创新科技人才” 职能素养蓝图, 建置流程经过相关职业职能分析、专家访谈、问卷验证、专家再确认， 最终获得六个关键职能素养群组与 38 项基本职能要素, 以及该职业所需之知识、技能与态度, 亦可成 为职业培训之重要基准, 以期加速智能机器人产业人才培育, 逐步改善人力结构, 创造产业价值链。 关键词: 人力资源開發，创新科技人才，职能模型，智能机器人，粤港澳大湾区 


\section{1. 前言}

《中国机器人产业发展报告(2019)》揭露全球各类 型智能机器人市场规模持续扩大, 处于快速增长时期 ${ }^{[1]}$ 。 随着全球新一轮科技和产业的蓬勃发展, 智能机器人是 一项涵盖多项领域整合性之产业, 被认为是推动中国经 济、科技与教育发展的关键产业之一 ${ }^{[1]}$ 。然而, Zhang 与 Zhang (2018) ${ }^{[2]}$ 研究指出中美两国的高端人才现状比较, 中国存在优秀的智能机器人, 人才在业界人数较少和结 构不合理的问题。由于智能机器人产业快速发展和行业 对人才需求量激增, 出现人才巨大缺口, 其次中国在技 术研发起步较欧美国家缓慢, 行业专家及人才不足, 专 利申请数量庞大而质量却并不高、缺乏关键核心技术和 完善的培训教育机制(Chen, Zhang, \& Wang, 2019) ${ }^{[3]}$ 。人 才不足实为发展智能机器人产业最大隐忧。因此, 引发 本研究回归人才培养专业知识本质, 探讨智能机器人产 业高端”创新科技人才”共同核心职能素养，以促进学校、 训练机构、产业可以快速培养所需的技术人才，以期提 供粤港澳大湾区(下述简称“大湾区”)相关产业及高校在 未来培育和养成人才教育与训练之参考依据。

本研究以大湾区智能机器人产业作为研究对象, 其 原因有三: 大湾区已成为中国全方位对外开放的重要经 贸地区, 具经济指标作用; 其次, 大湾区具有良好的产 业基础, 拥有全中国最大、最多的智能机器人园区, 且 已经形成高端装备制造、3C制造等为代表的产业集群; 其三, 大湾区内含丰富的人才资源, 和已经建立的高校 联盟及创新创业、职业教育等, 为智能机器人产业发展 不断注入动力。

\section{2. 文献探讨}

\section{1. 职能概念}

职能模型(competency model), 是指构成每一项工作 表现所应具备的职能群组。McClelland(1973) $)^{[4]}$ 认为以传 统智力测验来预测个体工作成就效果不彰, 智力测验的 内涵往往是学业表现的翻版, 与实际工作内容脱节, 于 是提出冰山模型(iceberg model), 把职能(competence)描 述为漂浮在海洋上的冰山, 裸露在水面上部分, 有基本 知识、基本技能, 属于外层表现部分, 对任职者基础素 质要求, 相对比较容易通过培训习得来改变和发展, 也 是人力资源工作者容易了解与测量部分; 水面下部分是 个体内在的、最不容易被评估和了解部分，它代表个体 有自己独特的思维方式和理念, 包括社会角色、自我形 象、特质、动机等, 这些特质有些是天生的, 有些是个 体成长环境因素所造成, 只有其主观能动性变化影响到 工作时, 其对工作的影响才会体现出来, 相对于水面上 的知识和技能而言, 这部分很难通过后天的培训得以形 成(Spencer \& Spencer, 1993) ${ }^{[5]}$ 。

\section{2. 职能研究现状}

Boyatzis $(2008)^{[6]}$ 认为职能就是找出一种让个体在 工作表现出高效能, 或出色的重要特性, 透过职能可以 用来预测某人在某项职务上表现的效能高低。近年来国 内外研究职能成果亦非常丰硕, 如 Lin, Wu 与 Tsai (2018) [7]研究专科护理师之职能分析, 提出未来之训练方向及 工作职能提升之策略。Ou 与 Yang (2018) ${ }^{[8]}$ 建构复合式 大楼物业管理经理人五个构面职能, 为管理职能、核心 职能、专业职能、一般职能及自我职能。Wu 与 Lin (2018) [9]以问卷调查法, 调查个案公司 179 名信息人员的职能 与工作绩效关系, 包括专业知识、专业技能、人际关系、 工作态度等四个职能构面。Fang (2017a, 2017b $)^{[10-11]}$ 针对 LED 照明产业初任工程师所需职能分析, 和风力机发电 产业工程师职能分析。Patanakul 与 Milosevic (2008) ${ }^{[12]}$ 探究项目经理应具备的职能模型。Sun, Shute, Stewart 等 (2020) $)^{[13]}$ 研究 CPS (Collaborative problem solving)职能模 型等。Hilburn, Ardis, Kornecki 与 Mead (2013) ${ }^{[14]}$ 建立软 件保障专业人员能力基础职能模型。

以上有关国内外职能相关研究虽多, 主要集中在中 高层管理、医护、技术性等领域，但对其新兴的智能机 器人产业高端人才核心职能研究目前尚未发现, 亦表示 人力资源学科工作者对此领域的研究尚未涉及, 特别是 智能机器人产业。目前大湾区智能机器人产业的高速发 展，高端创新科技人才供需匹配、培养方向、绩效考核 尚缺乏一套客观的衡量标准职能素养, 因此研究针对大 湾区智能机器人产业尝试发展所需的职能素养, 其研究 成果将对智能机器人产业在人才培养或绩效考核具有 一定的参考作用。

\section{3. 研究方法与设计}

研究针对大湾区智能机器人业者为样本, 邀请企业 内具有代表性资深工作人员及主管职人员为研究对象, 进行问卷填答, 以确保数据的信效度。问卷设计分为两 个部分，第一部分是询问职能素养题项，区隔有知识、 能力、态度三个部分; 第二部分是填答者个人基本信息, 包括性别、年龄、教育程度、工作年限、职务名称等。 采 Likert 的 5 点量表, 1-很不重要, 2-不重要, 3-普通, 4-重要, 5-很重要。

知识、能力、态度三个维度的问卷题项设计, 主要 是根据 Yu (2019) ${ }^{[15]}$ 的智能网联汽车行业研发人员职能 模型研究、Liu (2019) ${ }^{[16]}$ 的人工智能企业产品经理职能模 型构建研究、和 Sun (2018) ${ }^{[17]}$ 的云环境下 IT 管理者胜 任力模型实证研究, 等人的职能量表讨论、归纳而来的, 初步完成的题项有 58 个, 聚焦在知识维度有 22 个, 能 力维度有 23 项, 态度维度有 13 项。再经研究团队与业 界专家讨论, 考虑智能机器人产业”创新科技人才”, 强 调创新导向和思维方式、重视产品管理和团队协作, 后 剔除与”创新科技人才”关联性较低的职能素养要素，进 一步确定出 38 个职能正式问卷项目。 


\section{4. 研究发现与分析}

\section{1. 问卷填答对象}

研究针对大湾区智能机器人业者为样本, 邀请企业 内具有代表性资深工作人员及主管职人员为研究对象, 进行问卷填答。于 2019 年 12 月 8 日进行问卷发放, 共 114 份, 一星期后回收 107 份, 剔除填答不完全 4 份问 卷, 最终得到 103 份有效问卷, 有效率为 $96.26 \%$ 。 103 份问卷中, 男性 49 位(47.57\%), 女性 54 位(52.43\%)。 主要学历本科 68 位 $(66.02 \%)$, 专科 16 位 $(15.53 \%)$ 。年 龄主要在 25-30 岁 52 位(50.49\%), 31-40 岁 18 位(17.48\%) 之间, 工作年资 3-5 年人数最多, 说明在该产业里活跃 的人员大部分是中阶层级以上青年人。

\section{2. 因素分析与群聚命名}

本研究先行对问卷进行 KMO 和 Bartlett 球形检验, $\mathrm{KMO}$ 为 0.957 , Bartlett 球形卡方检验值为 4139.411, 且 Sig 值小于 0.010 , 说明问卷各题项间的关系是良好的 ${ }^{[41]}$ 。 问卷的 Cronbach's $\alpha$ 系数均在 0.832-0.969 之间具有较 好的信度(如表 1 所示)。通过主成分分析法并经最大方 差法正交旋转筛选分析, 共获得六个大于 1 的特征值, 其特征值分别为 $18.491 \% 、 16.228 \% 、 11.72 \% 、 11.449 \%$ 、 $10.691 \% 、 9.073 \%$, 累计率为 $77.652 \%$, 说明问卷具有良 好的结构效度。六个特征值如下说明:

专业素养能力: 因素 1 由 13 个题项因素形成, 其 中有 9 个题项因素属能力维度(可行性评估、测试验证、 设备常规维护、调机测试、创造力与创新、沟通能力、 程序编制能力、产品需求分析、风险控制能力), 4 个属 态度维度(团队合作、自信心、抗压性、适应力)。即智 能机器人是一项涵盖多领域的综合性产业, 从业者必须 具备多项跨领域专业能力, 根据设备组件特性规划可行 性测试流程与方案、针对应用环境找出优化模组参数、 选择恰当的算法设计程序构架、维护研发设备及工具的 完好。在协作时, 能与不同岗位同频交流和逻辑换位思 考能力。面对工作压力能够迅速调整、追求挑战并自我 改善, 根据任务目标不断学习成长。

科学应用能力: 因素 2 由 9 个题项因素形成, 其中 6 个题项因素属知识维度 (数理知识、编程能力、计算机 基础、数据分析、算法基础、工具基础知识), 3 个属能 力维度 (系统检验、资源协调、问题解决)。此着重科学 分析的数学、物理学、人工神经网络及计算机软件能力 的知识基础能力, 能熟练掌握并运用如 $\mathrm{C} / \mathrm{C}++$ 、Python、 Java、Matlab、Caffe 等工具, 协调并执行 AI 产品开发、 建立、检验及维护操作过程, 在遇到状况时能厘清问题, 运用系统化的方法, 提出解决方案。

逻辑理解能力: 因素 3 由 5 个题项因素形成, 其中 有 2 个题项因素属知识维度(统计学、分析能力), 3 个 属能力维度 (AI 技术理解力、 $\mathrm{AI}$ 行业理解力、系统规划)。 需具备 $\mathrm{AI}$ 行业知识、基本的 $\mathrm{AI}$ 概念和技术边界的基础
上，还需要有开放性、全面性的思维，在分析和解决复 杂问题时, 学习和理解因果推断, 通过不同的思维方式 把握问题，充分具体的评估系统限制范围从而制定规划。

组织管理能力: 因素 4 由 5 个题项因素形成, 其中 有 3 个题项因素属能力维度(成本概念、影响力、机械研 发与设计), 2 个属态度维度(组织承诺、计划与执行)。 需具备较强的组织协调及工作统筹的管理能力, 对工作 具有忠诚度和职业道德，能够有效管控项目收支、障碍 预测、实施过程，以专业的态度调动其他员工的工作热 情, 在充分利用资源的基础上合理分配任务, 制定计划 并按时执行贯彻。

表 1 智能机械人产业创新科技人才职能素养

\begin{tabular}{|c|c|c|c|}
\hline 构面 & 素养内涵 & $\begin{array}{l}\text { 因素载 } \\
\text { 荷系数 }\end{array}$ & $\begin{array}{c}\text { Cronbach's } \\
\alpha \text { 系数 }\end{array}$ \\
\hline \multirow{13}{*}{$\begin{array}{l}\text { 专业 } \\
\text { 素养 } \\
\text { 能力 }\end{array}$} & 适应力 & 0.833 & \multirow{13}{*}{0.969} \\
\hline & 团队合作 & 0.804 & \\
\hline & 抗压性 & 0.759 & \\
\hline & 自信心 & 0.741 & \\
\hline & 沟通能力 & 0.728 & \\
\hline & 产品需求分析 & 0.728 & \\
\hline & 测试验证 & 0.741 & \\
\hline & 调机测试 & 0.689 & \\
\hline & 风险控制 & 0.678 & \\
\hline & 可行性评估 & 0.609 & \\
\hline & 创造力与创新 & 0.623 & \\
\hline & 程序编制 & 0.601 & \\
\hline & 设备常规维护 & 0.586 & \\
\hline \multirow{9}{*}{$\begin{array}{l}\text { 科学 } \\
\text { 应用 } \\
\text { 能力 }\end{array}$} & 数理知识 & 0.970 & \multirow{9}{*}{0.939} \\
\hline & 编程能力 & 0.850 & \\
\hline & 计算机基础 & 0.810 & \\
\hline & 数据分析 & 0.758 & \\
\hline & 算法基础 & 0.683 & \\
\hline & 工具基础知识 & 0.675 & \\
\hline & 系统检验 & 0.664 & \\
\hline & 资源协调 & 0.563 & \\
\hline & 问题解决 & 0.636 & \\
\hline \multirow{5}{*}{$\begin{array}{l}\text { 逻辑 } \\
\text { 理解 } \\
\text { 能力 }\end{array}$} & $\mathrm{AI}$ 技术理解力 & 0.833 & \multirow{5}{*}{0.896} \\
\hline & AI 行业理解力 & 0.775 & \\
\hline & 统计学 & 0.771 & \\
\hline & 分析能力 & 0.579 & \\
\hline & 系统规划 & 0.540 & \\
\hline \multirow{5}{*}{$\begin{array}{l}\text { 组织 } \\
\text { 管理 } \\
\text { 能力 }\end{array}$} & 成本概念 & 0.750 & \multirow{5}{*}{0.942} \\
\hline & 组织承诺 & 0.714 & \\
\hline & 影响力 & 0.680 & \\
\hline & 计划与执行 & 0.606 & \\
\hline & 机械研发与设计 & 0.614 & \\
\hline 工业 & 工业焊接 & 0.634 & 0.897 \\
\hline
\end{tabular}




\begin{tabular}{|l|l|l|l|}
\hline \multirow{2}{*}{ 应用 } & 主要零件校核 & 0.768 & \multirow{2}{*}{} \\
\cline { 2 - 3 } & 工程图纸绘制 & 0.774 & \\
\hline 主动 & 行业背景 & 0.730 & \multirow{2}{*}{0.832} \\
\cline { 2 - 3 } 进取 & 快速学习 & 0.645 & \\
\cline { 2 - 3 } 精神 & 主动进取 & 0.685 & \\
\hline
\end{tabular}

工业应用能力: 因素 5 由 3 个题项因素形成, 主要 为能力维度 (工业焊接、主要零件校核、工程图纸绘制)。 需展现 AI 产业高端人才在工业制作中所需的工程实践 能力, 熟悉机器人结构, 能够运用 CAD、CAXA 或三维 软件设计产品的工程图纸、完成焊接结构的总体和细部 设计工作、对主要零件和部件进行精确的应力分析并修 正，有现场作业和处理装配的经验。

主动进取精神: 因素 6 由 3 个题项因素形成, 其中 有 1 个题项因素属知识维度(了解行业背景), 2 个属态 度维度(主动进取、主动学习)。在大数据、云计算背景 下, 互联网环境更迭飞速, AI 产业高端人才不仅需要具 备过硬的行业背景知识, 不拘于现有的产品设备, 还要 不断篗取新方法、新思想、新技术, 积极主动地进行深 度学习获得多领域的知识储备, 追求极致、保持创新思 维。

\section{5. 结论与建议}

\section{1. 结论}

本研究着眼于粤港澳大湾区, 人工智能领域的智能 机器人产业的”创新科技人才” 职能素养蓝图, 运用相关 职业职能分析、专家访谈、问卷验证、专家再确认, 最 终获得六个关键核心职能素养群组与 38 项基本职能要 素, 以及该职业所需之知识、技能与态度。除了探讨”创 新科技人才”专业人员的职能素养外, 亦期望进而减少 人才流动率及降低企业成本。研究藉由专家确认、问卷 验证, 使得六个职能素养群组能更切合实务上需求, 因 为, 并非每一种能力在某一种职缺中所占的百分比皆相 同。此外, 明确数值数据左证与支持, 可以减少人为决 策过程中所衍生之不科学性成份。至于”科学应用能力” 的”数理知识 $(0.970)$ "、“编程能力 (0.850)" 分析结果, 显 见是智能机器人产业所需求的职能素养能力, 符合目前 强调”创新科技人才”的理念。此外, 由于制程必须遵守 各种流程详细规范, 对于产品的生产须符合特定规格, 通常不允许轻易变更, 因此, 六个关键核心职能素养群 组中的”专业素养能力”、“逻辑理解能力”注重规则, 此 项职能素养亦有一定程度的重要性。

\section{2. 建议}

本研究旨在发展”创新科技人才”之职能素养基准, 以作为后续之职能导向训练课程发展的参考。在职能素 养的验证阶段所用之问卷, 在各题项填答人员可能不够
精确，尚有许多方法，如行为事例访谈、评估绩效等， 可用来作为判断适当职能素养的依据。本研究所发展之 职能素养，除了解”创新科技人才”职能素养外，可进一 步提供各项信息给人力资源管理主要活动参考, 如训练、 发展、绩效、薪资管理等, 再视需求针对落差较大之职 能项目安排训练计划。对后续研究者之建议, 可以针对 不同地区同产业的专业人员进行专业职能素养之研究, 以丰富产业之”创新科技人才”的专业职能素养研究。在 研究方法的改善与讨论, 可以再增加分析样本数量, 以 比较出不同职能素养差异。

\section{References}

[1] Chinese Institute of Electronics (2019). China Robot industry development report. Available at: https://www.chainnews.com/articles/185300551473.htm.

[2] Zhang, M.C., Zhang, Z. (2018) The Situation of artificial intelligence talents in China and its training methods. Modern Educational Technology, 28(8): 19-25.

[3] Chen, J., Zhang, Y. J., Wang, J. (2019) A comparative study on the development of AI industry between China and U.S.A. based on patent analysis. Journal of Intelligence, 38(1): 41-47.

[4] McClelland, D.C. (1973) Testing for competence rather than for intelligence. American Psychologist, 28(1): 1-14.

[5] Spencer, L.M., Spencer, S.M. (1993) Competence at work: Models for superior performance. New York: John Wiley \& Sons Inc.

[6] Boyatzis, R. E. (2008) Competencies in the 21st century. Journal of management development, 27(1): 5-12.

[7] Lin, S.C., Wu, M.L., Tsai, M.C. (2018) Research on the function of nurse practitioners. Journal of Health Management, 16(1): 65-73.

[8] Ou, H.W., Yang, M.L. (2018) Constructing the competency of property management managers. Journal of property management, $9(2): 15-30$.

[9] Wu, L.Y., Lin, H.C. (2019) The research on the relationship between the competence and job performance of information staff. Journal of Technology and Manpower Education, 5(4): 50-72.

[10] Fang, Y.S. (2017) An analysis of core competence for initial engineers for LED lighting industry. Science \& Technology Progress and Policy, 34(12): 36-42.

[11] Fang, Y.S. (2017) Identifying initial engineers competencies for the wind power industry. China Population, Resources and Environment, 27(5): 281-284. 
[12] Patanakul, P., Milosevic, D. (2008) A competency model for effectiveness in managing multiple projects. The Journal of High Technology Management Research, 18(2): 118-131.

[13] Sun, C., Shute, V. J., Stewart, A., Yonehiro, J., Duran, N., D'Mello, S. (2020) Towards a generalized competency model of collaborative problem solving. Computers \& Education, 143: 1-17.

[14] Hilburn, T., Ardis, M., Kornecki, A., Mead, N. R. (2013) Software assurance competency model. Software Engineering Institute.

[15] Yu, L.Q. (2019) Research on the competency model of $\mathrm{R}$ \& D personnel in the intelligent connected automotive industry. Shanghai Academy of Social Sciences, Shanghai.

[16] Liu, H. (2019) Research on the competency model construction of product manager of artificial intelligence enterprise. Anhui University, Hefei.

[17] Sun, X.N. (2018) Empirical research on competency model of IT managers in cloud environment. Jiangsu University of Science and Technology, Zhenjiang. 